\title{
METTALOTHIONEIN SENSING USING MOLECULARLY IMPRINTED POLYMERS COUPLED LASER ABLATION INDUCTIVELY COUPLED PLASMA-MASS SPECTROMETRY
}

\author{
${ }^{1}$ Kristyna ZEMANKOVA, ${ }^{1}$ Kristyna PAVELICOVA, ${ }^{1}$ Jaroslava BEZDEKOVA, ${ }^{1,2}$ Lukas NEJDL, \\ ${ }^{3}$ Marketa VEJVODOVA, ${ }^{3}$ Tomas VACULOVIC, ${ }^{1,2}$ Marketa VACULOVICOVA \\ ${ }^{1}$ Department of Chemistry and Biochemistry, Mendel University in Brno, Czech Republic, EU, \\ zemankova.kristyna@seznam.cz \\ ${ }^{2}$ Central European Institute of Technology, Brno University of Technology, Czech Republic, EU \\ ${ }^{3}$ Department of Chemistry, Faculty of Science, Masaryk University, Brno, Czech Republic, EU
}

https://doi.org/10.37904/nanocon.2020.3749

\begin{abstract}
In this work, we report a facile method for a detection of mettalothionein (MT) - potential biomarker of tumor diseases. Combination of laser ablation inductively coupled plasma coupled to mass spectrometric detection (LA-ICP-MS) with sample pretreatment by magnetic particles modified by molecularly imprinted polymers (Mag-MIP) enables selective and sensitive protein detection and quantification. After Mag-MIP MT extraction, elements specific for the protein (, $\mathrm{Zn}$ and $\mathrm{Cd}$ ) were detected in healthy pig skin and pig melanoma tissue using MeLiM (Melanoma-bearing-LibechovMinipig) model. It was found that levels of detected elements were significantly elevated in melanoma tissue compared to healthy skin. Currently, the investigation of MT dimerization in cancer progression is carried out taking advantage from easily controllable in vitro MT dimerization which is used for simple preparation of selective MIP extraction phase. Unlike other recognition elements, which are not always available for target analyte (e.g. antibodies), MIPs can be easily prepared as required. Moreover, magnetic particles as a MIP substrate enable elegant extraction approach and LA-ICP-MS provides extremely sensitive detection. Therefore, Mag-MIP-LA-ICP-MS is a unique cross-border combination of molecular and elemental analysis.
\end{abstract}

Keywords: Metallothionein, Molecularly imprinted polymers, Magnetic particles, LA-ICP-MS

\section{INTRODUCTION}

Metallothioneins (MTs) are a class of low molecular-weight (6 to $7 \mathrm{kDa}$ ) and cysteine-rich proteins. MTs are intracellular and ubiquitous in eukaryotes, and have specific structural characteristics giving them potent metal-binding and redox capabilities [1], due their high affinity to metals, MTs participate in the regulation of cellular metabolism of metals like zinc and copper, in detoxification of toxic metals like copper, cadmium and mercury, and in protection of cells against reactive oxygen species and alkylating agents [2]. MTs participate in the carcinogenic process, which include the generation of reactive oxygen species, oxidative DNA damage, genomic instability and others [3].

Malignant melanoma is one of the most dangerous and deadly forms of cancer, being responsible for $1.2 \%$ of all cancer deaths in the European Union. Malignant melanomas often go unnoticed, it is characterized by early metastasis and poor prognosis, and its incidence is increasing each year $[4,5]$. For immediate therapy and increase of survivors of melanoma, evaluation of histopathological and clinical parameters is important and mainly, the identification of potential biomarkers plays a critical role [6]. 
Molecularly imprinted polymers (MIPs) are synthetic materials with artificially generated recognition sites able to selectively interact with target molecules. Currently, MIPs are attracting widespread attention due to their properties such as flexibility, variability, high chemical and mechanical stability, relatively low cost and simplicity in preparation $[7,8]$. The use of nanomaterials - magnetic nanoparticles - in combination with MIPs provides large surface-to-volume ratio and well defined shape; moreover possibility of rapid separation of magnetic particles by the aid of an external magnet is favorable [9].

In this work, a method combining laser ablation inductively coupled plasma mass spectrometric detection (LA-ICP-MS) with sample pretreatment by magnetic particles modified by molecularly imprinted polymers (Mag-MIP) was developed. The suggested approach enables selective and sensitive protein detection and quantification, especially detection of MT. After Mag-MIP MT extraction, elements specific for the protein ( $\mathrm{S}, \mathrm{Zn}$ and $\mathrm{Cd}$ ) were detected as this information is essential for further development of effective and timely diagnostic tools for melanoma.

\section{MATERIALS AND METHODS}

\subsection{Materials and reagents}

Dopamine hydrochloride, Trizma $^{\circledR}$ base and acetic acid were purchased from Sigma-Aldrich (MO, USA) in ACS purity. MT from rabbit liver was obtained from Enzo Life Science (NY, USA). Dynabeads ${ }^{\mathrm{TM}}$ MyOne ${ }^{\mathrm{TM}}$ Silane magnetic particles and sodium tetraborate decahydrate were purchased from Thermo Fisher Scientific (MA, USA).

\subsection{Preparation of magnetic MIPs}

Briefly, $50 \mu \mathrm{l}$ of magnetic nanoparticles (MPs) $\left(40 \mathrm{mg} \cdot \mathrm{mL}^{-1}\right)$ were washed by $200 \mu \mathrm{L}$ of $20 \mathrm{mM}$ TRIS ( $\mathrm{pH}$ 8.5) for three times. MT (1 mg $\cdot \mathrm{mL}^{-1}$ ) was dissolved in $20 \mathrm{mM}$ TRIS ( $\mathrm{pH} \mathrm{8.5)} \mathrm{and} 400 \mu \mathrm{L}$ of solution was added to the washed MPs for preparation of Mag-MIPs. To prepare non-imprinted polymers (NIPs) used as a control, only $400 \mu \mathrm{L}$ of $20 \mathrm{mM}$ TRIS (pH 8.5) was added to the washed MPs. The mixtures were stirred for 1 hour and then $100 \mu \mathrm{L}$ of dopamine $\left(17.5 \mathrm{mg} \cdot \mathrm{mL}^{-1}\right)$ dissolved in $20 \mathrm{mM}$ TRIS $(\mathrm{pH} 8.5)$ was added and the reaction was continued overnight at room temperature. The product was collected using external magnetic field, supernatant was removed and discarded. The template was washed out three times by $200 \mu \mathrm{L}$ of $10 \%$ acetic acid and once by $200 \mu \mathrm{L}$ of MilliQ.

\subsection{Sample preparation}

After washing of Mag-MIPs and Mag-NIPs by $20 \mathrm{mM}$ TRIS (pH 8.5), $200 \mu \mathrm{L}$ of sample was added and mixture was left shaking for 2 hours. After supernatant removal, Mag-MIPs and Mag-NIPs were washed three times with $200 \mu \mathrm{L}$ of $20 \mathrm{mM}$ TRIS (pH 8.5) and samples were analyzed by LA-ICP-MS.

\subsection{LA-ICP-MS analysis}

LA-ICP-MS experiments were performed using a setup consisting of the laser ablation system UP213 (NewWave Research, USA) emitting laser radiation with a wavelength of $213 \mathrm{~nm}$. The ablated material was washed away using $\mathrm{He}\left(1.0 \mathrm{~L} \cdot \mathrm{min}^{-1}\right)$ from the ablation chamber (Supercell). Ar flow $\left(0.6 \mathrm{~L} \cdot \mathrm{min}^{-1}\right)$ was admixed into a flow of helium with the sample aerosol behind the ablation cell. Hence, the total gas flow was $1.6 \mathrm{~L} \cdot \mathrm{min}^{-1}$. This mixture was fed into a quadrupole ICP-(Q)MS spectrometer Agilent $7500 \mathrm{CE}$ (Agilent Technologies, Japan) equipped with a collision-reaction cell for suppressing possible polyatomic interferences. The CRC was utilized in collision mode with a He $(99.000 \%)$ flow rate of $2 \mathrm{~mL} \cdot \mathrm{min}^{-1}$. 


\section{RESULTS AND DISCUSSION}

The MIP-based separation procedure using MPs and following LA-ICP-MS analysis is schematically shown in Figure 1.

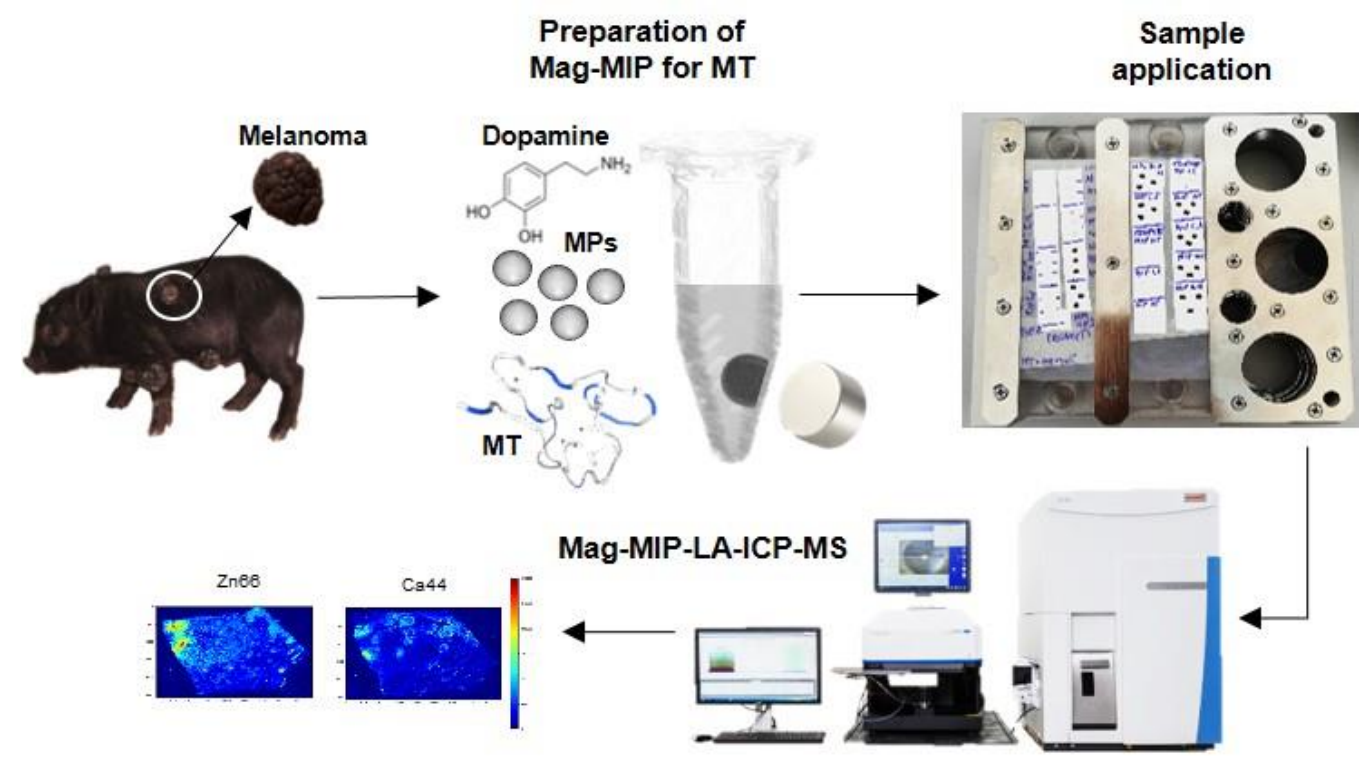

Figure 1 Schematic experimental workflow of Mag-MIP-LA-ICP-MS.

\subsection{Metal detection in MT by LA-ICP-MS}

Current analytical methods for MT detection include capillary electrophoresis, immunoassays, liquid chromatography, matrix-assisted laser desorption/ionization time-of-flight mass spectrometry and electrochemistry [10]. LA-ICP-MS is suitable for quantitative analysis of very low concentrations therefore; it was employed for quantitation of levels of metal ions present in MT.

In Figure 2, determined $\mathrm{Zn}$ levels are shown. The nonspecific sorption on polymeric layer without presence of cavities (NIP) was reasonably low and this background signal can be subtracted for quantification purposes.

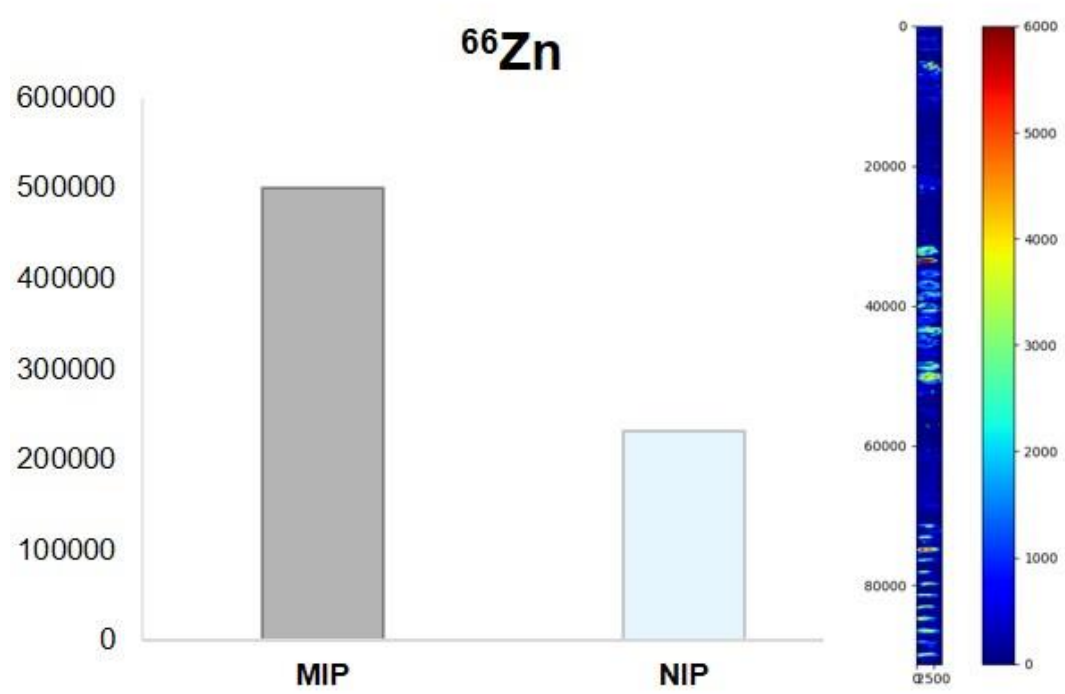

Figure 2 LA-ICP-MS analysis of ${ }^{66} \mathrm{Zn}$ levels after extraction of MT by Mag-MIP 


\subsection{Recognition of melanomas}

For evaluation of melanoma, analysis of clinical parameters is important as well as identification of potential biomarkers. MT is highly probably one of such markers.

In this study, the melanomas and healthy skin of melanoma-bearing minipigs (MeLiMs) were tested using the magnetic MIPs specific for MT and it was found that levels of detected elements were significantly elevated in melanoma tissue compared to healthy skin (Figure 3).

$66 \mathrm{Zn}$

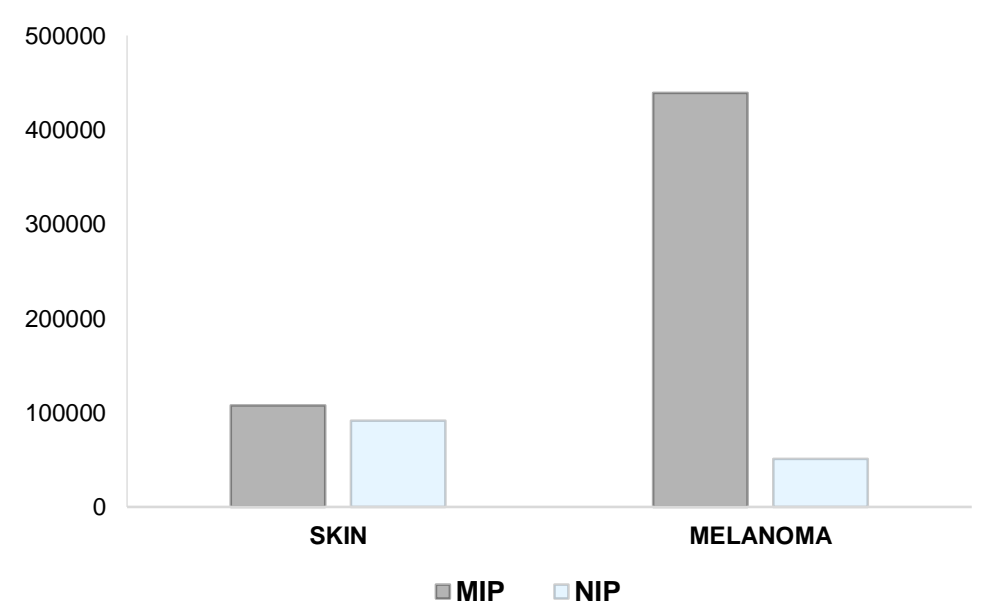

Figure 3 Levels of ${ }^{66} \mathrm{Zn}$ detected in MT isolated by Mag-MIP from healthy skin and melanoma of MeLiMs

\section{CONCLUSION}

In this work, power and variability of molecular imprinting technology in combination with high surface area of nanomaterials and magnetic properties of iron oxide creates an extremely effective tool for bioanalytical applications. Mag-MIP-LA-ICP-MS is a unique cross-border combination of molecular and elemental analysis. Mainly, the ${ }^{66} \mathrm{Zn}$ level was elevated in the majority of melanomas in comparison with healthy skin. This new approach enables the get information which is essential for further development of effective and timely diagnostic tools for melanoma detection.

\section{ACKNOWLEDGEMENTS}

\section{The research was financially supported by AF-IGA20120-IP059.}

\section{REFERENCES}

[1] GAD, S.C. Metallothionein. In: P. Wexler. Encyclopedia of Toxicology. Third Edition. Oxford: Academic Press, 2014, pp. 230.

[2] PALUMAA, P., et al. Metal binding of metallothionein-3 versus metallothionein-2: lower affinity and higher plasticity. Biochimica et Biophysica Acta (BBA) - Proteins and Proteomics. 2005, vol. 1747, no. 2, pp. 205211.

[3] SLUSSER, A., et al. Metallothionein isoform 3 expression in human skin, related cancers and human skin derived cell cultures. Toxicology Letters. 2015, vol. 232, no. 1, pp. 141-148. 
[4] FALLER, W.J., et al. Metallothionein 1E is methylated in malignant melanoma and increases sensitivity to cisplatin induced apoptosis. Melanoma Research. 2010, vol. 20, no. 5, pp. 392-400.

[5] KOPF, A.W., BART, R.S., RODRÍGUEZ-SAINS, R.S. Malignant Melanoma: A Review. ImmunoTargets and Therapy. 1977, vol. 3, no. 1, pp. 41-117.

[6] KRIZKOVA, S., et al. Microarray analysis of metallothioneins in human diseases-A review. Journal of Pharmaceutical and Biomedical Analysis. 2016, vol.117, pp. 464-473.

[7] SAMAH, N.A., et al. Molecularly imprinted polymer for the removal of diclofenac from water: Synthesis and characterization. Sci Total Environ. 2018, vol. 631-632, pp. 1534-1543.

[8] UZURIAGA-SÁNCHEZ, R.J., et al. Magnetically separable polymer (Mag-MIP) for selective analysis of biotin in food samples. Food Chem. 2016, vol. 190, pp. 460-467.

[9] ZHAO, Y., et al. Surface imprinted polymers based on amino-hyperbranched magnetic nanoparticles for selective extraction and detection of chlorogenic acid in Honeysuckle tea. Talanta. 2018, vol. 181, pp. 271277.

[10] MERLOS RODRIGO, M.A., et al. Fully automated two-step assay for detection of metallothionein through magnetic isolation using functionalized $\mathrm{y}-\mathrm{Fe} 2 \mathrm{O} 3$ particles. Journal of chromatography. $B$, Analytical technologies in the biomedical and life sciences. 2016, vol. 1039, pp. 17-27. 